\title{
Multimodal Prehabilitation in Patients with Non-Small Cell Lung Cancer Undergoing Anatomical Resection: Protocol of a Non- Randomised Feasibility Study
}

\author{
Charlotte Johanna Laura Molenaar ( $\nabla$ charlotte.molenaar@mmc.nl ) \\ Maxima Medisch Centrum https://orcid.org/0000-0003-2407-4197 \\ Erik Martin Von Meyenfeldt \\ Albert Schweitzer Ziekenhuis \\ Carlijn Tini Ireen de Betue \\ Albert Schweitzer Ziekenhuis \\ Goof Schep \\ Maxima Medisch Centrum \\ Magdolen Youssef El Soud \\ Maxima Medisch Centrum \\ Eric van Thiel \\ Albert Schweitzer Ziekenhuis \\ Nicky Rademakers \\ Maxima Medisch Centrum \\ Sanne Charlotte Hoornweg \\ Albert Schweitzer Ziekenhuis \\ Gerrit Dirk Slooter \\ Maxima Medisch Centrum \\ Frank van den Broek \\ Maxima Medisch Centrum \\ Geertruid Marie Heleen Marres \\ Maxima Medisch Centrum
}

\section{Study Protocol}

Keywords: Prehabilitation, preoperative intervention, enhanced recovery after thoracic surgery, non-small cell lung cancer, anatomical resection, lung surgery, functional capacity, physical conditioning, postoperative outcome, feasibility

Posted Date: August 13th, 2020

DOI: https://doi.org/10.21203/rs.3.rs-57399/v1

License: @ (1) This work is licensed under a Creative Commons Attribution 4.0 International License. Read Full License 


\section{Abstract}

\section{Background}

The preoperative period can be used to enhance a patient's functional capacity with multimodal prehabilitation and, consequently improve and fasten postoperative recovery. Especially non-small cell lung cancer (NSCLC) surgical patients may benefit from this intervention, since the affected and resected organ is an essential part of the cardiorespiratory fitness. Drafting a prehabilitation programme is a challenge, since many disciplines are involved, and time between diagnosis of NSCLC and surgery is limited. We designed a multimodal prehabilitation programme prior to pulmonary surgery and conduct a study to assess feasibility and efficacy of the programme. Publication of this protocol may help other health care facilities to implement such a programme.

\section{Methods}

The multimodal prehabilitation programme consists of an exercise programme, nutritional support, mental support, smoking cessation programme, patient empowerment and respiratory optimisation. In two Dutch teaching hospitals, 40 adult patients with proven or suspected NSCLC will be included. In a non-randomised fashion, 20 patients follow the multimodal prehabilitation programme and 20 will be assessed in the control group, according to patient preference. Assessments take place at four time points: baseline, week before surgery, six weeks postoperatively and three months postoperatively. Feasibility and efficacy of the prehabilitation programme will be assessed as primary outcomes.

\section{Discussion}

Since the time between diagnosis of NSCLC and surgery is limited, it is a challenge to implement a prehabilitation programme. This study will assess whether this is feasible and effective. The non-randomised fashion of the study might result in a selection bias. However, the control group is necessary, to put the prehabilitation group in perspective and to assess feasibility further. By publishing this protocol of our feasibility study, we aim to facilitate others to evaluate and implement a multimodal prehabilitation programme for surgical lung cancer patients.

\section{Trial registration}

This feasibility study is registered as NL8080 in the Netherlands Trial Register on the $10^{\text {th }}$ of October 2019, https://www.trialregister.nl/trial/8080. Secondary identifiers: CCMO (Central Committee on Research Involving Human Subjects) number NL70578.015.19, reference number of the Medical Ethical Review Committee of Máxima MC W19.045.

\section{Background}

Worldwide, lung cancer is the leading cause of cancer death and has the highest incidence amongst cancer types (1). The preferred curative treatment option for resectable non-small cell lung cancer (NSCLC) is anatomical resection combined with mediastinal lymph node dissection. However, surgery and postoperative complications contribute to significant morbidity. Patients with major pulmonary complications have poor long-term outcome, including a reduced disease free survival (2). Furthermore, (pulmonary) complications affect patient-centred outcomes and health-care costs (3).

In the past decades, the preoperative period has been used more and more to prepare patients for surgery. These (multimodal) prehabilitation programmes aim to enhance functional capacity, prepare patients for treatment and consequently, improve outcome. Several systematic reviews and meta-analyses on the effect of various preoperative interventions prior to lung cancer surgery have recently been published, reporting a reduction in postoperative (pulmonary) complications and length of hospital stay (4-7). In addition to these benefits, patients deemed unfit for surgery, based on their pulmonary function test and exercise test results, could potentially become a surgical candidate with prehabilitation (8).

The first official guidelines for enhanced recovery after lung surgery by the Enhanced Recovery After Surgery (ERAS ${ }^{a}$ ) Society and the European Society of Thoracic Surgeons (ESTS) strongly recommend prehabilitation for patients with borderline lung function or exercise capacity (9).

To date, delaying surgery for patients with lung cancer to facilitate preparation with prehabilitation cannot be recommended, because cancer could potentially progress $(4,8)$. The Dutch national guideline for the treatment of NSCLC states that $80 \%$ of patients should have been operated on within two weeks after completion of the diagnostic phase (10). This is monitored by the Dutch Institute for Clinical Auditing (DICA) through the compulsory Dutch Lung Cancer Audit - Surgery (DLCAs), which records and benchmarks the percentage of operations 
performed within three weeks from the multidisciplinary team meeting confirming diagnosis (11). The window of opportunity prior to surgery to optimise the patient's condition is therefore limited, yet meaningful interventions seem not impossible, as was demonstrated in a recently published randomised controlled trial with a multimodal prehabilitation programme in a home-based setting of only two weeks (12).

With our multicentre, non-randomised study we will evaluate the feasibility and efficacy of a supervised high-intensity multimodal prehabilitation programme for surgical NSCLC patients in the Dutch healthcare system. By publishing the protocol of our study we aim to accommodate colleagues to implement a similar programme in their own facilities more easily, and possibly adjust it to use for other patient populations as well. Publication of this protocol enables us to discuss the programme more thoroughly, than when reported simultaneously with the final results of our study. Besides, this publication will contribute to transparency in the conduction of studies.

\section{Methods}

\section{Study design and setting}

Our study is a non-randomised feasibility study, approved by the Medical Ethical Review Committee of Máxima MC (MMC) (reference number: W19.045) and the Institutional Review Board of the Albert Schweitzer Hospital (ASz). Patient recruitment has started January 2020 and was temporarily interrupted due to the Corona Virus epidemic. The two Dutch teaching hospitals (MMC and ASz) have expertise regarding prehabilitation programmes for oncological surgery and enhanced recovery after thoracic surgery.

For this protocol, the reporting standards of the SPIRIT (Standard Protocol Items: Recommendations for Interventional Trials) checklist, adapted to the CONSORT (Consolidated Standards of Reporting Trials) extension for pilot and feasibility studies was used as guidance (13).

\section{Participants}

All consecutive patients meeting the inclusion criteria are asked to participate in the multimodal prehabilitation programme. Patients are deemed eligible when $\geq 18$ years of age, with pathologically confirmed or suspected NSCLC and an indication for anatomical lung resection. Furthermore, a straightforward preoperative work-up is needed to ensure patients can enter the programme directly after diagnosis in order to use the window of opportunity between diagnosis and surgery as efficient as possible. Also, the ability to provide written informed consent is needed for inclusion. Eligible patients not willing to participate in the multimodal prehabilitation programme are asked to participate in the control group. The control group provides insight in the course of functional capacity perioperatively and enables comparison with the prehabilitation group.

Patients are excluded in case of a contra-indication for training, renal insufficiency (defined as estimated glomerular filtration rate (eGFR) $<60 \mathrm{ml} / \mathrm{min} / 1.73 \mathrm{~m}^{2}$ ) or participation in other trials that may interfere. Referred patients from another hospital with the diagnostic phase already completed, are excluded as well, for logistical reasons, travel distance and the probability that these factors may affect feasibility of the programme. When a contra-indication for training becomes apparent during baseline assessment, the subject is excluded from participation and will be replaced. Furthermore, in case the postoperative pathology results do not confirm the diagnosis NSCLC, the patient will be replaced as well. A total of 40 patients with confirmed NSCLC will be included in the study in both centres; per centre ten patients in the prehabilitation group and ten in the control group. All replaced, screened non-eligible and non-consenting patients are logged.

The sample size for this feasibility study is based on a previously performed pilot study in colorectal cancer patients (14). Patients are selected and informed about the study during a visit to their treating pulmonologist in the outpatient clinic and informed consent is obtained by a member of the research team during an intake prior to baseline assessment. The expected inclusion period will be approximately two years.

The SPIRIT derived figure 1 displays an overview of the time points, assessments and recorded variables in our feasibility study.

\section{Assessments}

Both groups are assessed at baseline, the week before surgery, six weeks postoperatively and three months postoperatively. The intensity of the exercises in the multimodal prehabilitation programme (discussed in the "interventions" section) in the intervention group is individualised, based on the patient's baseline assessments.

Physical status

Patients undergo an assessment by a physiotherapist to determine functional capacity, and are requested to fill in a questionnaire estimating physical condition. 
The questionnaire to estimate physical condition (FitMáx@) is used to determine the maximum oxygen uptake expressed as V02max. This questionnaire is developed by one of the authors with an expertise in exercise physiology (G. Schep) and is validated in 700 subjects varying form healthy to suffering from lung disease, heart disease or cancer. Preliminary results pointed out that the questionnaire had a correlation of 0.93 with VO2max determined with the Cardiopulmonary Exercise Test (CPET), the gold standard to determine functional capacity. Results will be published in the near future and a translation in English will become available before the end of the current study. The questionnaire is sent to patients by email at baseline, prior to surgery and six weeks as well as three months postoperatively.

Additionally, the physiotherapist determines functional capacity using the 6-minute walk test (6MWT), steep ramp test (SRT) and indirect one-repetition maximum (1-RM). The tests are completed at baseline, the week before surgery and six weeks postoperatively. The walking distance in meters during the 6MWT is determined by counting the number of times a patient walks back and forth on a straight 30 meters track during six minutes. Patients are encouraged and receive feedback according to a standardised schedule (according to the American Thoracic Society). Patients are allowed to use any walking device, if necessary (15). With the SRT, functional capacity is determined as maximum short exercise capacity (MSEC) on a calibrated bicycle ergometer. The test starts with three minutes of warming up at zero Watt, followed by an increase of resistance by 25 Watt per ten seconds while maintaining the revolutions per minute between 70 and 80 . The incremental phase continues until exhaustion occurs, defined as $<60$ revolutions per minute (16). The MSEC is expressed as total Watt achieved until exhaustion. To determine strength, 1-RM is the golden standard. In this study, the indirect 1-RM, defined as the weight a person can lift at least two and maximum ten times, is used because the 1-RM can be strenuous and may cause injuries. The 1-RM is calculated using the Bryzcki equation: 1-RM = (used weight/(1.0278-(0.0278*number of repetitions) (17).

To monitor the compliance to the out-of-hospital exercises (described in the "interventions" section) in the intervention group and monitor physical activity in the control group, an accelerometer (PAM AM 300, Pam private company, Oosterbeek, the Netherlands) is provided to participants in both groups for the duration of the study. Patients wear the accelerometer from the baseline assessment until three months after surgery, during daytime from getting out of bed until going to bed.

\section{Nutritional status}

Prior to the initial consultation by the dietitian, only the prehabilitation group completes a three-day dietary diary to estimate energy and protein intake.

To assess nutritional status, the following measurements are performed at baseline and the week prior to surgery in both groups. Weight and height are measured to calculate the body mass index (BMI) and the Patient-Generated Subjective Global Assessment (PG-SGA) is used to screen for malnutrition $(18,19)$. The PG-SGA is completed at baseline and the week prior to surgery. To determine body composition, a standardised bio-electrical impedance analysis (BIA) with a single frequency 50 kilohertz impedance meter (Bodystat 500, Bodystat, Isle of Man, United Kingdom) is performed. Different equations are used to calculate fat free mass (FFM), according to BMI and the presence of Chronic Obstructive Pulmonary Disease (COPD): Kyle when BMl< 30 (20), Horie with a BMI $\geq 30$ (21) and Rutten in case of COPD (22). Finally, the hand grip strength is determined with a hand-held hydraulic dynamometer (Jamar, Patterson Medical, Warrenville, Illinois, United States of America). The measurement is repeated three times, the highest result of both hands is recorded.

\section{Mental status}

The psychological assessment consists of the Hospital Anxiety and Depression Scale (HADS) (23), consisting of a seven-item subscale to assess complaints of anxiety and depression, and the Insomnia Severity Index (ISI) (24), assessing sleeping disturbances. In the prehabilitation group only, during a consultation with a clinical psychologist the results of these questionnaires are discussed. Since the score of the HADS is not expected to change in a limited period as in this study, it is conducted only at baseline. The ISI is completed at baseline, the week prior to surgery and three months after surgery.

\section{Quality of life; assessing_patient empowerment}

Quality of life is assessed at baseline, six weeks and three months after surgery, using the following questionnaires sent by email:

- EQ-5D-5L (Euroqol Group, 5-level general health-related quality of life questionnaire $(25,26)$ );

- EORTC QLQ-C30 (European Organisation for Research and Treatment of Cancer; quality of life of cancer patients $(27,28)$ );

- EORTC QLQ-LC13 (European Organisation for Research and Treatment of Cancer; quality of life of cancer patients - lung cancer specific (28, 29)). 
Additionally, a patient satisfaction questionnaire assessing all items of the programme is drafted by the research team and is sent by email to both groups the week prior to surgery and three months postoperatively.

\section{Respiratory status}

Pulmonary functioning is assessed according to standard of care. Additionally, the maximal inspiratory pressure (MIP) is measured with the MicroRPM (Respiratory Pressure Meter, Care Fusion, San Diego, California, United States of America) in order to determine the intensity of the inspiratory muscle training, which will be elaborated further in the "interventions" section. The MIP is measured three times, aiming for a difference less than $5 \%$. The highest pressure is recorded and used for the programme. The MIP is determined at baseline, the week before surgery and six weeks postoperatively.

\section{$\underline{\text { Cost-effectiveness }}$}

Finally, to estimate the cost-effectiveness of the multimodal prehabilitation programme, a shortened institute for Medical Technology Assessment (iMTA) questionnaire (30) is used, consisting of the iMTA Productivity Cost Questionnaire (iPCQ) and the iMTA Medical Consumption Questionnaire (iMCQ). Patients are requested to fill out the iPCQ and iMCQ at baseline and three months after surgery.

\section{Interventions}

The prehabilitation group starts with the multimodal prehabilitation programme after the baseline assessment. The programme ends the week before surgery. The multimodal prehabilitation programme contains six pillars: exercise programme, nutritional support, mental support, a smoking cessation programme, patient empowerment and respiratory optimisation (disease specific optimisation).

\section{Exercise programme}

The exercise programme consists of in-hospital, supervised training on three non-consecutive days per week, for about 60 minutes, consisting of high-intensity interval training (HIIT) endurance training and strength exercises. Patients are supervised by a physiotherapist, specialised in oncology and preferably train in peer groups if permitted by the periodicity of inclusion of subjects and existing Corona Virus Disease 2019 measures. The intensity of the exercise programme is individualised based on the baseline assessment, and continuously evaluated by the physiotherapist during the programme.

The HIIT is performed on a bicycle ergometer and consists of two series of eight repetitions of uninterrupted cycling. One repetition consists of 30 seconds of strain, set on $65 \%$ of the MSEC measured with the SRT, followed by 30 seconds of rest being $30 \%$ of the MSEC. Intensity of the HIIT is monitored and adjusted, if necessary, according to the following: if a patient scores $\leq 11$ on the Borg scale (31), the intensity is increased by $5 \%$; intensity is decreased by $5 \%$ when the Borg score is $\geq 15$ or when a patient is not able to complete the eight repetitions.

The supervised strength exercises are performed on fitness devices and the intensity is based on the 1-RM calculation at baseline. The intensity starts with $65 \%$ of 1 -RM in week one, increases to $70 \%$ in week two and $75 \%$ in week three. Training consists of two series of ten repetitions of the following exercises, targeting major muscle groups: lateral pull down, leg press, chest press and low row.

Finally, patients are instructed by the physiotherapist to perform out-of-hospital exercises - like walking or cycling, according to patient preference - with low to moderate intensity, on the remaining four days of the week for at least 60 minutes per day.

\section{Nutritional support}

Nutritional status is optimised through nutritional counselling by a registered dietitian. Based on the baseline assessment, the dietitian provides a tailored dietary advice, including energy and protein requirements. Energy intake is based on the calculated energy expenditure. The recommended daily protein intake is 1.9-2.2 grams of protein/kilogram FFM (as determined with BIA). When FFM cannot be calculated, actual body weight is used with a daily protein recommendation of 1.5-1.8/ kilogram.

All patients in the prehabilitation group receive a high-quality protein powder supplement containing whey and casein proteins, providing ten grams of essential amino acids - of which two to three grams of Leucine - per portion of 30 grams (Refitß-TMP-90-Shake, Friesland Campina Domo, Amersfoort, the Netherlands). Patients are instructed to ingest 30 grams of supplement within one hour after the in-hospital supervised training and daily one hour before going to sleep.

Additionally, vitamin D and multivitamin supplements are provided for daily use. Vitamin D dosage depends on age, gender, skin type and sun exposure and is based on the guideline of the Health Council of the Netherlands (32). 
Mental support consists of a 45-minute explorative consultation by a clinical psychologist. During this consult, burden of the disease and accompanied treatment as well as the patient's coping strategies are assessed, and when indicated empowerment or psychoeducation is provided. The clinical psychologist determines whether follow-up sessions are needed during the perioperative phase and/or referral to a psychiatrist is indicated and acts accordingly. Furthermore, breathing and relaxation techniques, assisted with audio are provided to the patients.

\section{$\underline{\text { Smoking status }}$}

If applicable, patients are offered to follow a smoking cessation programme. This consists of counselling and nicotine replacement therapy and is outsourced to a specialised institute in the Netherlands (SineFuma private company, Breda, the Netherlands) (33).

\section{Patient empowerment}

To maximise patient empowerment, participants are optimally informed and educated by the research team about the prehabilitation programme, the surgical care pathway and their own contribution. An information booklet containing information on all interventions of the programme is handed out; a logbook is provided to register all study related activities.

Respiratory optimisation (disease specific optimisation).

Disease specific optimisation is directed at the affected organ system; the respiratory system in the case of lung cancer. During the inhospital supervised sessions, patients perform inspiratory muscle training (IMT). An IMT device (Philips-Respironics Threshold IMT, Philips, Eindhoven, the Netherlands) is provided to the patient and intensity is based on the MIP assessment at baseline. The training starts at $40 \%$ of the MIP and increases to at least 50\%. The patient receives instructions from the physiotherapist how to perform the exercise at home. Training is performed twice daily for 15 minutes. Additionally, breathing and sputum clearance techniques are taught by the physiotherapist prior to surgery.

\section{Outcome measures}

\section{Primary outcomes}

The primary outcomes of this study are feasibility of the prehabilitation programme and functional capacity. Feasibility is defined as $\geq 80 \%$ protocol adherence (per intervention) of at least $80 \%$ of the participants in the prehabilitation group. To determine feasibility, we will collect the following data: number of eligible patients, number of patients enrolled, number of drop outs, number of attended supervised training sessions (per patient), patient's logging booklet (number of out-of-hospital low to moderate training, recorded intake of nutritional supplements) and accelerometer data. Functional capacity is determined at baseline, the week prior to surgery and six weeks postoperatively. Change over time compared to baseline within and between groups will be determined.

\section{Secondary outcomes}

The following secondary outcomes will be studied:

- Programme evaluation as a whole and per component (based on compliance, patient satisfaction and experience of the research team);

- Effect on clinical outcome: 30-day mortality, length of hospital stay, 30-day complication rate expressed as the comprehensive complication index (34) and readmission rate. This will be compared to the control group and a historical Dutch cohort as recorded in the DLCAs (11);

- Cost-effectiveness of the prehabilitation programme;

- Effectiveness of the programme on nutritional and mental status, smoking cessation and quality of life compared to baseline and control group;

- Correlation between V02max reported through the physical condition questionnaire (FitMax@) and as determined with the SRT and if applicable, CPET.

\section{Data handling}

With the use of an electronic case report form (eCRF), a database was built in Castor electronic data capture (EDC) (35). Castor EDC complies with Title 21 of the Code of Federal Regulations Part 11, General Data Protection Regulation (GDPR) and is fully ISO (International 
Organization for Standardization) 27001, 27002 and 9001 certified. When a new patient is registered in Castor EDC, patient codes are automatically generated, complying with the GDPR. Most of the data from the assessments are entered directly into the eCRF. The remaining data is entered by members of the research team in both centres. To guarantee the quality of this study, monitoring will be executed by an independent party appointed by the sponsor. All data saved in Castor EDC and source files will be stored for a period of 15 years.

\section{Statistical analysis}

Because of the sample size of the group, descriptive data will be presented as median (interquartile range and/or range). Categorical data will be presented as numbers and percentages. Results of validated questionnaires will be calculated and missing data will be handled using the instructions from the original authors. Missing data which will be used in the definition of feasibility, will be considered as "not performed".

Feasibility will be descriptively evaluated (group level and patient level), both dichotomously (yes/no) and as percentage of the total number of offered interventions.

Comparative outcomes, such as functional capacity, between the different test moments will be compared within the group participating in the multimodal prehabilitation programme and within the control group with the Friedman test and between groups with the Mann-Whitney $\mathrm{U}$ test. Nonparametric tests will be used because of the small sample size. Dichotomous data will be compared between groups with Chisquare test.

\section{Discussion}

Since many disciplines are involved and time is limited, multimodal prehabilitation is a logistic challenge for any hospital. It is widely studied in patients with colorectal cancer and deemed feasible and effective $(14,36,37)$. However, the treatment interval for NSCLC is shorter than for colorectal cancer in the Netherlands due to tumour biology.

However, especially the lung cancer population may particularly benefit from prehabilitation. The pulmonary system is one of the determinants of cardiorespiratory fitness, a measure interpreted as the reflection of total body health (38), as well as the organ system affected by NSCLC. Furthermore, the lung is the organ that is resected during surgery, thereby further compromising cardiorespiratory fitness in itself. Although improving cardiorespiratory fitness preoperatively will not prevent a decrease of functional capacity postoperatively, prehabilitation may limit the extent.

This protocol is a result of an extensive multidisciplinary project. Two clinical groups with expertise in perioperative recovery optimisation developed a protocol based on experience and available evidence, combining various interventions within a short timeframe. In order to make a patient friendly programme, all disciplines need to optimise and harmonise planning.

To our knowledge, only one randomised controlled trial with a multimodal prehabilitation programme in a NSCLC population has been published, however, this concerned a non-supervised home-based programme with less interventions compared to our study (12). In the Netherlands, we experience a growing interest in prehabilitation prior to surgery, yet to date there are no formal public prehabilitation programmes available for lung surgery patients.

The results of prehabilitation are promising thus far in other cancer types (39-41). Since one of the primary aims of our study is to determine the feasibility - the main perceived barrier for application - of our multimodal prehabilitation programme, a study design based on randomisation was deemed suboptimal. Although a similar pilot study in patients with colorectal cancer did not find a better baseline functional capacity in the prehabilitation group compared to the control group (14), a selection bias may occur in our study. The control group in our study will put the prehabilitation group in perspective, since this enables us to determine the "normal" course in the perioperative period using data from the assessments and accelerometers. Additionally, studying the control group may help assess feasibility further.

In conclusion, with this study we aim to examine if an extensive, mainly in-hospital multimodal prehabilitation programme is feasible in the limited preoperative period of maximum three weeks, as determined by our national guideline. Furthermore, we aim to study whether patientoptimisation can be achieved. By publishing this protocol, we provide a detailed description of the programme to improve transparency. Hopefully, this may help other hospitals to shorten the process of multidisciplinary set up and implement a multimodal prehabilitation programme more easily.

\section{List Of Abbreviations}


(Indirect) 1-RM: (indirect) one-repetition maximum

6MWT: 6-minute walk test

ASz: Albert Schweitzer Hospital

BIA: bio-electrical impedance analysis

BMI: body mass index

BSc: Bachelor of Science

Castor EDC: Castor electronic data capture

CCMO: Central Committee on Research Involving Human Subjects

CONSORT: Consolidated Standards of Reporting Trials

COPD: Chronic Obstructive Pulmonary Disease

CPET: cardiopulmonary exercise test

DICA: Dutch Institute for Clinical Auditing

DLCAs: Dutch Lung Cancer Audit - Surgery

eCRF: electronic case report form

eGFR: estimated glomerular filtration rate

EORTC QLQ-C30: European Organisation for Research and Treatment of Cancer; quality of life of cancer patients module

EORTC QLQ-LC13: European Organisation for Research and Treatment of Cancer; quality of life of cancer patients lung cancer module

EQ-5D-5L: Eurogol Group, 5-level general health-related quality of life questionnaire

ERAS: Enhanced Recovery After Surgery

ESTS: European Society of Thoracic Surgeons

FFM: fat free mass

GDPR: General Data Protection Regulation

HADS: hospital anxiety and depression scale

HIIT: high-intensity interval training

iMCQ: iMTA Medical Consumption Questionnaire

IMT: inspiratory muscle training

iMTA: institute for Medical Technology Assessment

iPCQ: iMTA Productivity Cost Questionnaire

ISI: insomnia severity index

ISO: International Organization for Standardization

MD: Medical Doctor

MIP: maximal inspiratory pressure 
MMC: Máxima MC

MSc: Master of Science

MSEC: maximum short exercise capacity

NSCLC: non-small cell lung cancer

PG-SGA: patient-generated subjective global assessment

PhD: doctor of philosophy

SPIRIT: Standard Protocol Items: Recommendations for Interventional Trials

SRT: steep ramp test

V02max: maximal oxygen uptake

\section{Declarations}

\section{Ethics approval and consent to participate:}

The study has been reviewed and approved by the accredited Medical Research Ethics Committee of MMC (reference: W19.045) and by the institutional review board of ASz. This feasibility trial is registered as NL8080 in the Netherlands Trial Register on $10^{\text {th }}$ of October 2019 . All patients sign written informed consent to participate according to Good Clinical Practice guidelines. When applicable, protocol amendments will be submitted to the Medical Research Ethics Committee of MMC for approval.

\section{Trial sponsor:}

both hospitals contribute equally to the conduct of the feasibility study and are responsible for their own financing.

\section{Consent for publication:}

not applicable.

\section{Availability of data and materials:}

not applicable.

\section{Competing interests:}

The authors declare that they have no competing interests.

\section{Funding:}

FrieslandCampina provides the protein supplements for this study. ASz uses a subsidy received to support scientific research, to finance aspects of this trial. The funding sources had no efforts in the design of this study, nor will they have a role in the collection, analysis, interpretation of data or completion of this trial.

\section{Authors' contributions:}

All (co-)authors contributed to the design of the original protocol. CM en EvM drafted the first version of the manuscript. All authors reviewed and contributed to the completion of this manuscript, and approved the final version.

\section{Acknowledgements:}

all authors are member of the Dutch Prehab Lung Research Group. Also the following co-authors are members of the research group: Loes van de Voort, BSc (physiotherapist MMC); Chris de Jongh, MSc (physiotherapist FYSIOOOO); Cathrin van Erven, MSc (dietitian MMC); Mirjam Staffeleu - Noodelijk (dietitian MMC); Els Driessen, MSc (clinical psychologist ASz); Marieke van de Wal, MSc (clinical psychologist MMC); Netty de Graaff, MSc (master advanced nursing practice); Anouk van Limpt, MSc (nurse specialist lung cancer MMC); Maaike Scholten-Bakker, PhD (pulmonary function department ASz); Rosaline van den Berg, PhD (scientific consultant, Science Office ASz). 
this manuscript is based on the study protocol version 5.0, dated the $2^{\text {nd }}$ of December 2019.

\section{Roles and responsibilities:}

Both centres have a principal investigator (G. Marres in ASz and G. Slooter in MMC) to be responsible for the accurate conduction and progress of this study. They may delegate tasks to any member of the research team. The Clinical Trial Centre in Maastricht is responsible for the data monitoring in MMC. An independent, trained research assistant from MMC will monitor data in ASz.

\section{References}

1. Bray F, Ferlay J, Soerjomataram I, Siegel RL, Torre LA, Jemal A. Global cancer statistics 2018: GLOBOCAN estimates of incidence and mortality worldwide for 36 cancers in 185 countries. CA: A Cancer Journal for Clinicians. 2018 Nov;68(6):394-424.

2. Wang S, Li X, Li Y, Li J, Jiang G, Liu J, et al. The long-term impact of postoperative pulmonary complications after video-assisted thoracic surgery lobectomy for lung cancer. Journal of thoracic disease. 2017 Dec;9(12):5143-52.

3. Templeton R, Greenhalgh D. Preoperative rehabilitation for thoracic surgery. Current opinion in anaesthesiology. 2019 Feb;32(1):23-8.

4. Cavalheri V, Granger C. Preoperative exercise training for patients with non-small cell lung cancer. The Cochrane database of systematic reviews. 2017 Jun 7;;6:CD012020.

5. Kendall F, Oliveira J, Peleteiro B, Pinho P, Bastos PT. Inspiratory muscle training is effective to reduce postoperative pulmonary complications and length of hospital stay: a systematic review and meta-analysis. Disability and Rehabilitation. 2018 Apr 10;440(8):86482.

6. Rosero ID, Ramirez-Velez R, Lucia A, Martinez-Velilla N, Santos-Lozano A, Valenzuela PL, et al. Systematic Review and Meta-Analysis of Randomized, Controlled Trials on Preoperative Physical Exercise Interventions in Patients with Non-Small-Cell Lung Cancer. Cancers (Basel). 2019 July 05;11(7):10.3390/cancers11070944.

7. Steffens D, Beckenkamp PR, Hancock M, Solomon M, Young J. Preoperative exercise halves the postoperative complication rate in patients with lung cancer: a systematic review of the effect of exercise on complications, length of stay and quality of life in patients with cancer. Br J Sports Med. 2018 March 01;52(5):344,098032. Epub 2018 Feb 1.

8. Mahendran K, Naidu B. The key questions in rehabilitation in thoracic surgery. Journal of thoracic disease. 2018 Apr;10(Suppl 8):S92430.

9. Batchelor TJP, Rasburn NJ, Abdelnour-Berchtold E, Brunelli A, Cerfolio RJ, Gonzalez M, et al. Guidelines for enhanced recovery after lung surgery: recommendations of the Enhanced Recovery After Surgery (ERAS $®$ ) Society and the European Society of Thoracic Surgeons (ESTS). European Journal of Cardio-Thoracic Surgery. 2019 Jan 1;;55(1):91-115.

10. National guideline non-small cell lung cancer [Internet]. [cited 4th of August 2020]. Available from: https://richtlijnendatabase.nl/richtlijn/niet_kleincellig_longcarcinoom/organisatie_van_zorg/maximaal_aanvaardbare_wachttijden.html.

11. Dutch Institute for Clinical Auditing [Internet]. [cited June 2019]. Available from: https://dica.nl/dlca/home.

12. Liu Z, Qiu T, Pei L, Zhang Y, Xu L, Cui Y, et al. Two-Week Multimodal Prehabilitation Program Improves Perioperative Functional Capability in Patients Undergoing Thoracoscopic Lobectomy for Lung Cancer: A Randomized Controlled Trial. Anesth Analg. 2019 July 23.

13. Thabane L, Lancaster G. A guide to the reporting of protocols of pilot and feasibility trials. Pilot Feasibility Stud. 2019 February 28;5:37,8. eCollection 2019.

14. van Rooijen SJ, Molenaar CJL, Schep G, van Lieshout, R H M A, Beijer S, Dubbers R, et al. Making patients fit for surgery: introducing a four pillar multimodal prehabilitation program in colorectal cancer. Am J Phys Med Rehabil. 2019 May 13.

15. ATS statement: guidelines for the six-minute walk test. American journal of respiratory and critical care medicine. $2002 \mathrm{Jul}$ 1,;166(1):111-7.

16. Meyer K, Samek L, Schwaibold M, Westbrook S, Hajric R, Beneke R, et al. Interval training in patients with severe chronic heart failure: analysis and recommendations for exercise procedures. Medicine and science in sports and exercise. 1997 Mar;29(3):306-12.

17. Reynolds J, Gordon T, Robergs R. Prediction of one repetition maximum strength from multiple repetition maximum testing and anthropometry. Journal of Strength and Conditioning Research. 2006 Aug;20(3):584-92.

18. Ottery FD. Definition of standardized nutritional assessment and interventional pathways in oncology. Nutrition. 1996 January 01;12(1 Suppl):15. 
19. Sealy MJ, Hass U, Ottery FD, van der Schans, C P, Roodenburg JLN, Jager-Wittenaar H. Translation and Cultural Adaptation of the Scored Patient-Generated Subjective Global Assessment: An Interdisciplinary Nutritional Instrument Appropriate for Dutch Cancer Patients. Cancer Nurs. 2018 December 01;41(6):450-62.

20. Kyle UG, Schutz Y, Dupertuis YM, Pichard C. Body composition interpretation: Contributions of the fat-free mass index and the body fat mass index. Nutrition. 2003;19(7):597-604.

21. Horie LM, Gonzalez MC, Torrinhas RS, Cecconello I, Waitzberg DL. New Specific Equation to Estimate Resting Energy Expenditure in Severely Obese Patients. Obesity. 2011 May;19(5):1090-4.

22. Rutten EP, Spruit MA, Wouters EF. Critical view on diagnosing muscle wasting by single-frequency bio-electrical impedance in COPD. Respir Med. 2010 January 01;104(1):91-8.

23. Zigmond AS, Snaith RP. The hospital anxiety and depression scale. Acta Psychiatr Scand. 1983 June 01;67(6):361-70.

24. Bastien $\mathrm{CH}$, Vallieres A, Morin CM. Validation of the Insomnia Severity Index as an outcome measure for insomnia research. Sleep Med. 2001 July 01;2(4):297-307.

25. Herdman M, Gudex C, Lloyd A, Janssen M, Kind P, Parkin D, et al. Development and preliminary testing of the new five-level version of EQ-5D (EQ-5D-5L). Qual Life Res. 2011 December 01;20(10):1727-36.

26. Euroqol Group [Internet]. [cited June 2019]. Available from: www.euroqol.org.

27. Aaronson NK, Ahmedzai S, Bergman B, Bullinger M, Cull A, Duez NJ, et al. The European Organization for Research and Treatment of Cancer QLQ-C30: a quality-of-life instrument for use in international clinical trials in oncology. J Natl Cancer Inst. 1993 March 03;85(5):365-76.

28. European Organisation for Research and Treatment of Cancer [Internet]. [cited June 2019]. Available from: www.eortc.org.

29. Bergman B, Aaronson NK, Ahmedzai S, Kaasa S, Sullivan M. The EORTC QLQ-LC13: a modular supplement to the EORTC Core Quality of Life Questionnaire (QLQ-C30) for use in lung cancer clinical trials. EORTC Study Group on Quality of Life. Eur J Cancer. 1994;30A(5):635-42.

30. Bousema JE, Dijkgraaf MGW, Papen-Botterhuis NE, Schreurs HW, Maessen JG, van der Heijden, E H, et al. MEDIASTinal staging of nonsmall cell lung cancer by endobronchial and endoscopic ultrasonography with or without additional surgical mediastinoscopy (MEDIASTrial): study protocol of a multicenter randomised controlled trial. BMC Surg. 2018 May 18;18(1):27-6.

31. Achttien RJ, Staal JB, Merry A, et al. KNGF-richtlijn Hartrevalidatie. 2011.

32. Vitamin D guideline, Health Council of The Netherlands [Internet]. [cited June 2019]. Available from: https://www.gezondheidsraad.nl/documenten/adviezen/2012/09/26/evaluatie-van-de-voedingsnormen-voor-vitamine-d.

33. SineFuma [Internet]. [cited June 2019]. Available from: http://www.sinefuma.com/nl.

34. Slankamenac K, Graf R, Barkun J, Puhan M, Clavien P. The Comprehensive Complication Index: A Novel Continuous Scale to Measure Surgical Morbidity. Annals of Surgery. 2013 Jul;258(1):1-7.

35. Castor EDC [Internet]. [cited June 2019]. Available from: https://www.castoredc.com/.

36. Trepanier M, Minnella EM, Paradis T, Awasthi R, Kaneva P, Schwartzman K, et al. Improved Disease-Free Survival After Prehabilitation for Colorectal Cancer Surgery. Ann Surg. 2019 July 15.

37. Scheede-Bergdahl C, Minnella EM, Carli F. Multi-modal prehabilitation: addressing the why, when, what, how, who and where next? Anaesthesia. 2019 Jan;74(S1):20-6.

38. Ross R, Blair SN, Arena R, Church TS, Despres JP, Franklin BA, et al. Importance of Assessing Cardiorespiratory Fitness in Clinical Practice: A Case for Fitness as a Clinical Vital Sign: A Scientific Statement From the American Heart Association. Circulation. 2016 December 13;134(24):e653-99.

39. Minnella EM, Awasthi R, Bousquet-Dion G, Ferreira V, Austin B, Audi C, et al. Multimodal Prehabilitation to Enhance Functional Capacity Following Radical Cystectomy: A Randomized Controlled Trial. Eur Urol Focus. 2019 June 08.

40. Ploussard G, Almeras C, Beauval JB, Gautier JR, Garnault V, Fremont N, et al. A combination of enhanced recovery after surgery and prehabilitation pathways improves perioperative outcomes and costs for robotic radical prostatectomy. Cancer. 2020 July 08.

41. Swaminathan N, Kundra P, Ravi R, Kate V. ERAS protocol with respiratory prehabilitation versus conventional perioperative protocol in elective gastrectomy- a randomized controlled trial. Int J Surg. 2020 July 30.

\section{Figures}




\begin{tabular}{|c|c|c|c|c|c|c|}
\hline & \multicolumn{6}{|c|}{ STUDY PERIOD } \\
\hline TIMEPOINT & $-t_{1}$ & $\begin{array}{c}\mathbf{0} \\
\text { baseline }\end{array}$ & $\begin{array}{c}t_{1} \\
\text { Week before } \\
\text { surgery }\end{array}$ & Surgery & $\begin{array}{c}t_{2} \\
6 \text { Weeks } \\
\text { postoperatively }\end{array}$ & $\begin{array}{c}t_{3} \\
\text { 3 Months } \\
\text { postoperatively }\end{array}$ \\
\hline \multicolumn{7}{|l|}{ ENROLMENT } \\
\hline Eligibility screen & $\mathrm{x}$ & & & & & \\
\hline Informed consent & $\mathrm{x}$ & & & & & \\
\hline Allocation & & $\mathrm{x}$ & & & & \\
\hline \multicolumn{7}{|l|}{ INTERVENTIONS } \\
\hline \multicolumn{7}{|l|}{$\begin{array}{r}\text { Multimodal prehabilitation } \\
\text { Exercise progromme }\end{array}$} \\
\hline $\begin{array}{r}\text { Nutritionol support } \\
\text { Mentol support } \\
\text { Smoking cessation progromme } \\
\text { Patient empowerment } \\
\text { Respirotory optimisation } \\
\end{array}$ & & & & & & \\
\hline Control & \multicolumn{6}{|c|}{ No intervention } \\
\hline \multicolumn{7}{|l|}{$\begin{array}{l}\text { ASSESSMENTS \& VARIABLES } \\
\text { (control and prehabilitation group) }\end{array}$} \\
\hline \multicolumn{7}{|l|}{ Physical status } \\
\hline $\begin{array}{r}\text { Physiotherapist } \\
\text { SRT, 6MWT, indirect 1-RM } \\
\end{array}$ & & $\mathrm{x}$ & $\mathrm{x}$ & & $\mathrm{x}$ & \\
\hline Physical condition questionnaire* & & $\mathrm{x}$ & $\mathrm{x}$ & & $\mathrm{x}$ & $\mathrm{x}$ \\
\hline Accelerometer export & & & $\mathrm{x}$ & & $\mathrm{x}$ & $\mathrm{x}$ \\
\hline \multicolumn{7}{|l|}{ Nutritional status } \\
\hline Height & & $\mathrm{x}$ & & & & \\
\hline Weight \& BMl & & $\mathrm{x}$ & $\mathrm{x}$ & & & $\mathrm{x}$ \\
\hline$B I A$, hand grip strength, $P G-S G A^{*}$ & & $\mathrm{x}$ & $\mathrm{x}$ & & & \\
\hline $\begin{array}{c}\text { Three-day dietary diary } \\
\text { Oniy in prehabilitation group }\end{array}$ & & $\mathrm{x}$ & & & & \\
\hline \multicolumn{7}{|l|}{ Mental status } \\
\hline HADS* $^{*}$ & & $\mathrm{x}$ & & & & \\
\hline$|S|^{*}$ & & $\mathrm{x}$ & $\mathrm{x}$ & & & $\mathrm{x}$ \\
\hline \multicolumn{7}{|l|}{ Smoking status } \\
\hline Check with patient & & $\mathrm{x}$ & $\mathrm{x}$ & & $\mathrm{x}$ & $\mathrm{x}$ \\
\hline \multicolumn{7}{|l|}{ Patient empowerment } \\
\hline EQ-5D-5L, EORTC QLQ-C3O\&-LC13* & & $\mathrm{x}$ & & & $\mathrm{x}$ & $\mathrm{x}$ \\
\hline Patient satisfaction questionnaire* & & & $\mathrm{x}$ & & & $\mathrm{x}$ \\
\hline \multicolumn{7}{|l|}{ Respiratory status } \\
\hline MIP & & $\mathrm{x}$ & $\mathrm{x}$ & & $\mathrm{x}$ & \\
\hline \multicolumn{7}{|l|}{ Cost-effectiveness } \\
\hline${ }^{P} P C Q, M^{\prime} Q^{*}$ & & $\mathrm{x}$ & & & & $\mathrm{x}$ \\
\hline
\end{tabular}

Research and Treatment of Cancer, quality of life of cancer patients - lung cancer specific module; EORTC QLQ-C30, European Organisation

for Research and Treatment of Cancer, quality of life of cancer patients module; EQ-5D-5L, general health-related quality of life

questionnaire; HADS, hospital anxiety and depression scale; iMCO, institute for Medical Technology assessment (iMTA) Medical

Consumption Questionnaire; Indirect 1-RM, indirect 1-repetition maximum; IPCQ iMTA Productivity Cost Questionnaire; ISI, insomnia

severity index; MIP, maximal inspiratory pressure; PG-SGA, patient-generated subjective global assessment; SRT, steep ramp test. "All

questionnaires are sent by e-mail.

\section{Figure 1}

SPIRIT derived overview of time points, assessments and recorded variables. 